\title{
Arkadia via SNON enables NODAL-SMAD2/3 signaling effectors to transcribe different genes depending on their levels
}

\author{
Jonathon M. Carthy ${ }^{1, \dagger}$, Marilia Ioannou ${ }^{1 \dagger}$ and Vasso Episkopou ${ }^{1 *}$ \\ ${ }^{1}$ Imperial College London, Brain Sciences, Hammersmith Hospital Campus. \\ *Correspondence to: vasso.episkopou@imperial.ac.uk \\ $\dagger$ These authors contributed equally
}

\begin{abstract}
How cells assess levels of signaling and select to transcribe different target genes depending on the levels of activated effectors remains elusive. High NODAL-signalling levels specify anterior/head, lower specify posterior, and complete loss abolishes anterior-posterior patterning in the mammalian embryo. Here we show that cells assess NODAL-activated SMAD2 and SMAD3 (SMAD2/3) effector-levels by complex formation and pairing each effector with the corepressor SNON, which is present in the cell before signaling. These complexes enable the E3ubiquitin ligase Arkadia (RNF111) to degrade SNON. High SMAD2/3 levels can saturate and remove SNON, leading to derepression and activation of a subset of targets (high targets) that are highly susceptible to SNON repression. However, low SMAD2/3 levels can only reduce SNON preventing derepression/activation of high targets. Arkadia degrades SNON transiently only upon signaling exposure, leading to dynamic signaling-responses, which most likely initiate
\end{abstract}


level-specific cell-fate decisions. Arkadia-null mouse embryos and Embryonic Stem Cells (ESC) cannot develop anterior tissues and head. However, SnoN/Arkadia, double-null embryos and ESCs are rescued confirming that Arkadia removes SNON, to achieve level-dependent cell-fates

One Sentence Summary: Signaling intensity induces equivalent degradation of a transcriptional repressor leading to level-dependent responses.

\section{Introduction}

NODAL is a morphogen and a member of the transforming-growth-factor-beta (TGF- $\beta$ )-family ligand(1). It is transduced via C-terminal phosphorylation of SMAD2 and SMAD3 class of transcriptional effectors (SMAD2/3; hereafter refers to signaling-activated forms). SMAD2/3 complex with SMAD4 and translocate to the nucleus where they bind to DNA to initiate transcription(2-5). SMAD2/3 target-gene specificity is regulated by interactions with other transcription partner-factors present in the cell $(4,6)$. However, genetic experiments in mouse(7) and gain of function experiments in Xenopus and zebrafish $(1,8)$ show that during gastrulation different NODAL-signaling levels (intensities) activate distinct set of targets to establish various cell-fates along the anterior-posterior axis of the embryo. However, mechanisms that allow cells to assess NODAL-signaling levels and activate level-specific transcriptional responses remained $\operatorname{unknown}(9)$.

Gastrulation in mice is initiated by delamination of the epiblast epithelium at the prospective posterior end, followed by elongation of the delamination front towards the center of the embryo leading to Primitive Streak (PS) formation(10). Cells express Nodal only when they ingress 
within the PS, and turn it off when they migrate out laterally specified into precursors of specific tissues (germ layers)(7). In addition, these migrating precursors possess information about the prospective position that they will occupy along the anterior posterior axis of the embryo.

Gradual reduction of the SMAD2/3 effector-alleles using genetics in mouse causes loss of cellfates starting with those that derive from the elongating front of the PS (anterior-PS). Embryos with more advanced loss of effector-allele lose additional cell fates that derive from more posterior PS such as somites(11). These experiments suggest that graded NODAL-signaling is responsible for patterning the PS precursors with anterior-PS depending on maximum levels. Several NODAL targets operating during development have been identified however, it remained unclear whether they are activated by high or low NODAL signaling.

We previously showed that the RING-domain E3 ubiquitin ligase, RNF111 (Arkadia), is essential for the formation of anterior-/head tissues, but not of posterior, by a mechanism that depends on NODAL(12-14). Complementary experiments in Xenopus animal-cap assays showed that anterior cell fates are induced by co-injection of Nodal and Arkadia mRNAs but not by the same amount of Nodal or Arkadia injected alone, confirming that Arkadia potentiates NODAL signaling(13). Biochemical and cell culture experiments showed that Arkadia enhances signaling by targeting for degradation (a) the inhibitory SMAD7(15), which attenuates signaling mainly at the receptor level $(16,17)$, and (b) the nuclear co-repressors SNON and $\operatorname{SKI}(18,19)$, which bind to SMAD2, SMAD3 or SMAD4 and suppress their transcriptional responses(20). Notably, Arkadia has been shown to degrade SNON only when it is bound to SMAD2 or SMAD3(18). Whether the above substrates mediate the function of Arkadia in the embryo had not been determined. 
Here we show that Ark-/- embryos, which lack anterior/head structures, are rescued by genetic removal of SnoN, but not of Smad7, while embryos with single deletions of either SnoN (SnoN-/) or Smad7 (Smad7-/-) are normal. Similarly, Ark-/- ESCs cannot differentiate towards anterior cell-fates and exhibit defective activation of a subset of SMAD2/3-targets, but these are rescued in double-null Arkadia and SnoN ([Ark/SnoN]-/-) ESCs. We also show that Arkadia degrades the SNON-SMAD2 complex to achieves derepression within the first hour of signaling, and that SNON reduction is transient and proportionate to SMAD2/3 levels and derepression. Collectively, the above data reveal how cells assess NODAL-signaling levels and convert each of them to specific gene-expression patterns and cell fates.

\section{Results}

\section{Arkadia removes SNON to induce anterior in ESCs and embryos}

Cell-fates derived from the anterior-PS include the anterior definitive endoderm (ADE); the node, which is a structure forming at the end of the fully elongated PS after ADE migration; and the node-derived mesendoderm (ME), which is a cord-like structure extending along the midline joining the node with the ADE(7). ESCs differentiate towards ADE-ME with Activin-A (Activin; NODAL-like ligand) treatment(21). To address whether SNON is the substrate that mediates Arkadia's function we used WT and Ark-/- ESC lines derived from mouse embryos that carry the Hex-Gfp transgene, an ADE-ME reporter(22). WT ESC-lines (n=3) differentiate normally towards ADE-ME with Activin, while none of the Ark-/- lines (n=3) do so (Fig. 1A). However, deletion of SnoN via CRISPR/Cas9 in Ark-/- ESCs [SnoN/Ark]-/-, (n=10 clones; Fig S1A), rescues their ability to differentiate towards ADE-ME (Fig 1a). We confirmed the ADEME differentiation by quantitative PCR (qPCR) using the definitive endoderm marker Cxcr4(23), the ME marker Foxa2(24)(Fig 1B), and by western blot with GFP antibody (Fig. 
S1B). SnoN-/- ESC lines (n=3) differentiate normally towards ADE-ME (Fig. S1C and S1D), excluding the possibility that loss of SNON leads to excessive ADE-ME differentiation and it is responsible for the rescue observed in $[S n o N / A r k]-/-$ cells.

In the embryo the brain/head is induced by ADE and the adjacent ME domain (prechordal plate)(25), which migrate from the anterior-PS across and opposite, where they induce anterior neuroectoderm (brain) on the overlaying ectoderm layer(25). To address whether removal of SnoN from Ark-/- embryos can rescue their phenotype, we used mice, homozygous for $\operatorname{Skil}^{t m 2 S p w}(26)$, which are viable and fertile. We confirmed that these embryos are indeed SnoNnull (SnoN-/-) using whole-mount in-situ hybridization (WISH) with SnoN probe (Fig. S2A-C). The absence of an embryonic phenotype in SnoN-/- mice is puzzling however, we found that SKI, the close homologue of SNON, is upregulated in SnoN-/- embryos (Fig. S2E) suggesting compensation. Furthermore, using WISH with the ADE marker(27) Cerberus (Cer) we did not find any obvious difference in size or timing of ADE formation in SnoN-/- embryos (Fig. S2D). SNON is one of several feedback mechanisms that regulate fluctuations of NODAL-signaling intensity. Together these mechanisms increase the robustness of signaling regulation and most likely prevent dysregulation and phenotypes in the absence of SNON in vivo.

Consistent with the rescue that we observed in ESCs differentiation, [SnoN/Ark]-/- embryos also exhibit rescue of ADE, node-ME development along with midline and brain/head formation (Fig. 1C) with high penetrance $(>88 \%)$. Arkadia also degrades the NODAL-signaling inhibitor SMAD7(15), which mainly mediates the degradation of the TGF $\beta$-signaling receptors $(16,17)$ and therefore functions differently than SNON and SKI. Smad7-/- (28) mice, do not exhibit patterning defects during gastrulation and embryos develop normally, with some being born and surviving to adulthood. We generated [Smad7/Ark]-/- embryos and found that none was rescued 
as they are indistinguishable from the phenotype of Ark-/- embryos (Fig. 1D). Therefore, SNON, and not SMAD7, is the major substrate that mediates Arkadia's function during gastrulation.

Fig. 1

A

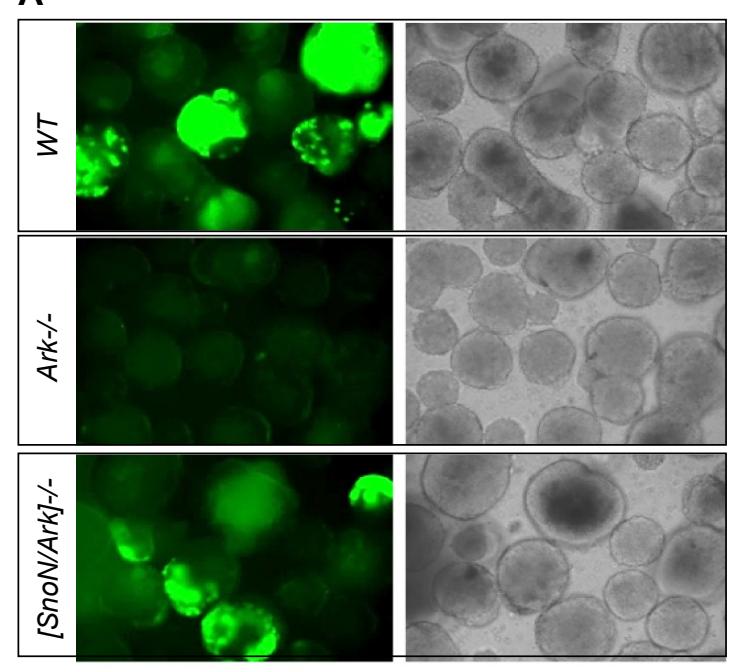

B

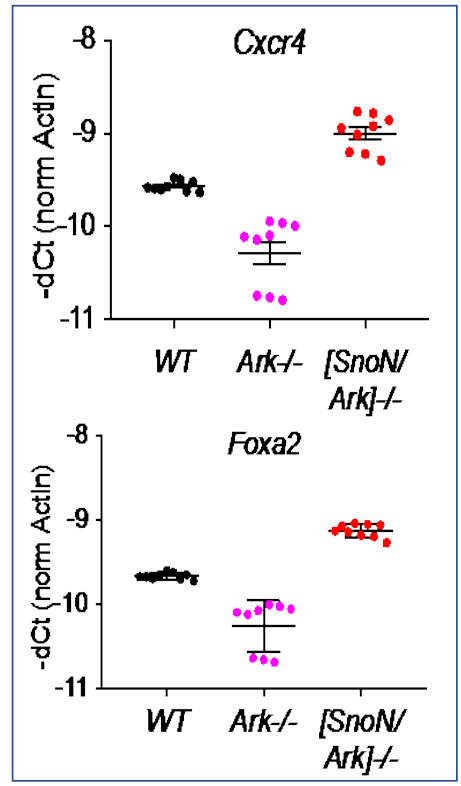

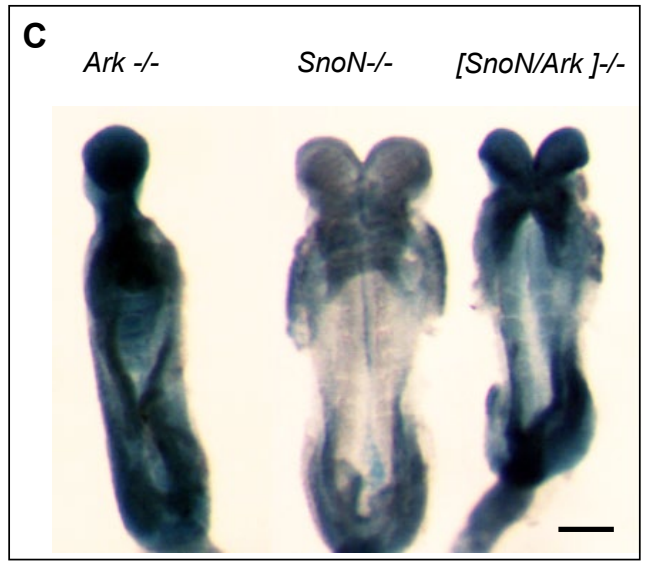

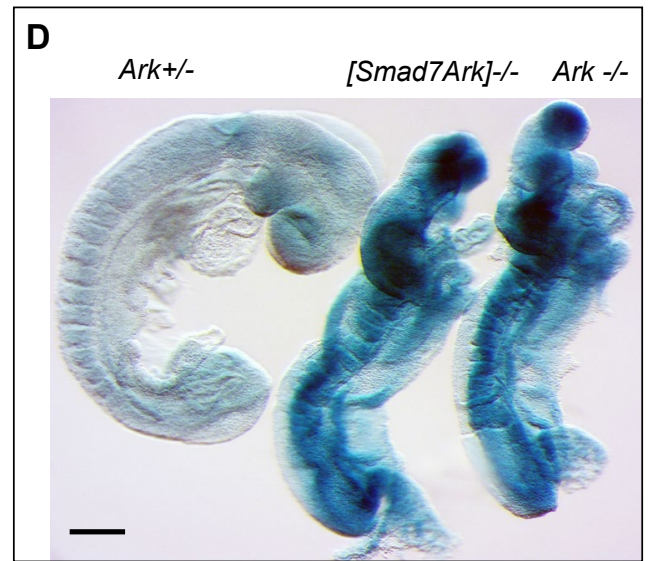

Fig.1: Arkadia removes SNON in ESCs and embryos. (A) Hex-Gfp ESCs differentiated towards ADE. Fluorescence (left) and bright field (right) images. (B) QPCR expression analysis of ADE/ME markers. (C) X-gal stained 6-8-somite E8.5 embryo from [SnoN-/-;Ark+/-]x[SnoN/-;Ark+/-] cross. Out of 63 embryos E8.5-9 analyzed, 18 were [SnoN/Ark]-/-, of which 16 seemed fully and 2 partially rescued ( $88 \%$ rescue). The rest of the embryos were of the parental genotype 
and were normal. (D) X-gal stained E9.5 embryos (>10 somites) from [Smad7+/-;Ark+/]x[Smad7+/-;Ark+/-] cross. Out of 207 embryos analyzed, 48 (23\%) exhibited Arkadia-null phenotype of which $15(7.3 \%)$ were $[$ Smad7/Ark]-/- (fewer than expected due to early resorptions). The rest of the embryos were single or double heterozygous and looked normal. SnoN-null and Ark-null alleles express $\beta$-galactosidase via their endogenous(12). Genotypes are as indicated; scale bar is $200 \mu \mathrm{m}$. 
We examined the extend of the rescue in [SnoN/Ark]-/- embryos using WISH with various makers: Cer probe showed that [SnoN/Ark]-/- embryos form ADE comparable to the $W T$ and SnoN-/- embryos while Ark-/- do not (Fig. 2A). Six3 (29) showed that [SnoN/Ark]-/-, WT and SnoN-/- embryos develop forebrain while Ark-/- do not (Fig. 2B). Both FoxA2(24) (Fig. 2C) and Shh(30) markers showed that unlike Ark-/-, [SnoN/Ark]-/-, WT and SnoN-/- embryos, form full length midline all the way to the brain (Fig 2d). The above embryo analysis confirms rescue of anterior-PS tissue development in [SnoN/Ark]-/- embryos. Shh marks also the hindgut endoderm(11) revealing that it forms in Ark-/- embryos (arrows in Fig. 2D) most likely because it derives from posterior- and not anterior-PS. However, [SnoN/Ark]-/- pups (n>50 litters) are not born indicating that later development cannot proceed in the absence of both Arkadia and SNON. We cannot exclude the possibility that SKI also mediates the function of Arkadia but to lesser extent. The most likely explanation for the above results is that a subset of NODALSMAD2/3 target genes responsible for the anterior-PS cell-fates are suppressed by SNON and depend on Arkadia and signaling for derepression and subsequent activation. 
Fig. 2
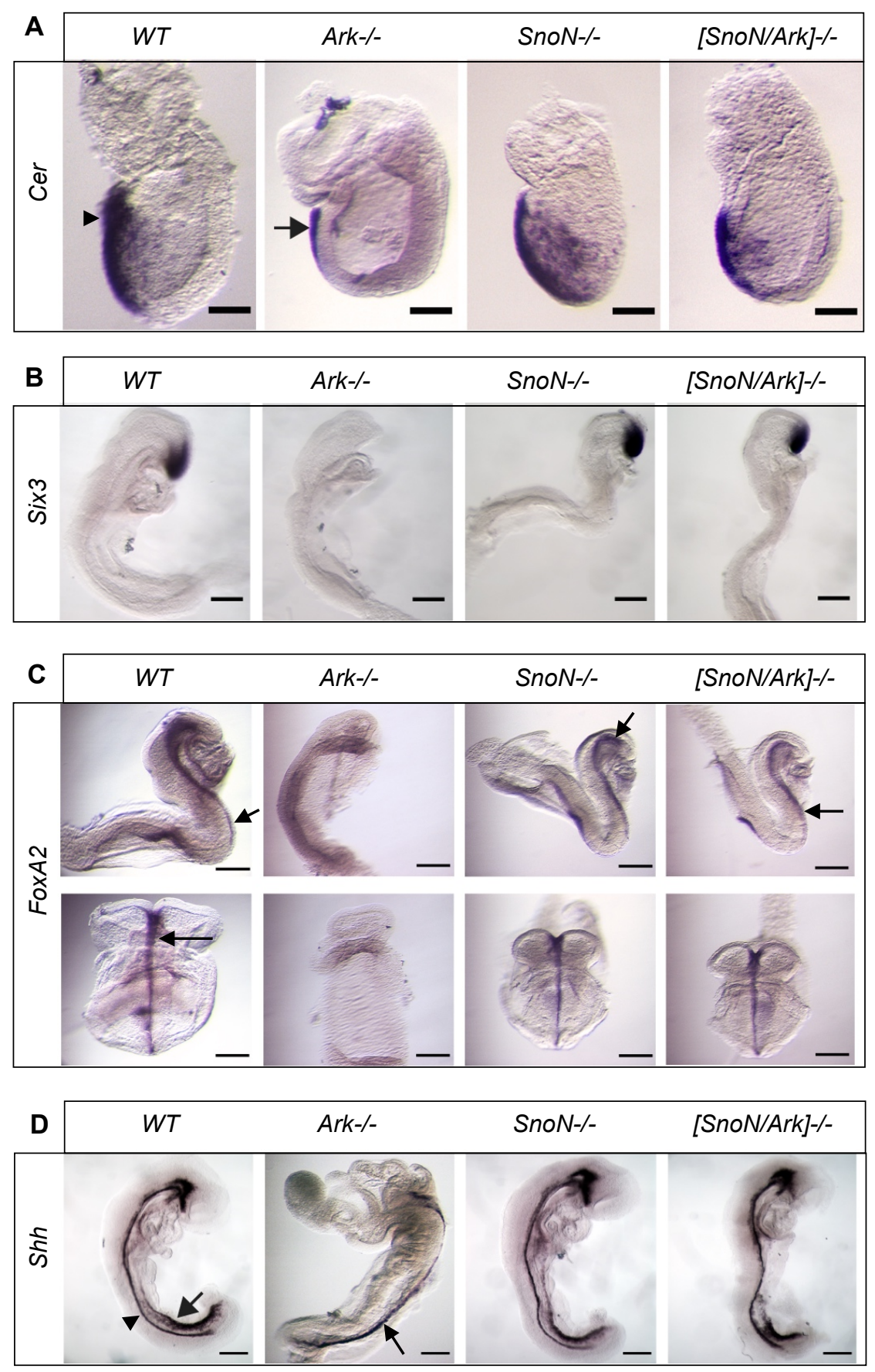

Fig. 2: Arkadia functions via SNON in the embryo. WISH with markers as indicated: (A)

E7.5 embryos stained with Cer are shown with anterior on the left and scale bar $100 \mu \mathrm{m}$. Ark-/-

embryos are known to lack ADE (arrowhead) but retain the extraembryonic anterior visceral 
endoderm (AVE) domain (arrow)(14). (B) Lateral view of E8.5 embryos stained with Six3 is shown. Six3 expression is rescued in [SnoN/Ark]-/- embryos. (C) Foxa2 stained E8.5 embryos with lateral (top) and ventral/frontal (bottom) views are shown with arrows pointing the ME. (D) Lateral view of Shh stained E9.5 embryos are shown with arrows pointing the hindgut and arrowheads the ME. Scale bar: $100 \mu \mathrm{m}(\mathbf{A}) ; 200 \mu \mathrm{m}$ (B-D). 


\section{Arkadia derepresses SNON-repressed genes}

To identify Arkadia-dependent NODAL target genes we used two different WT and two Ark-/ESC-lines (undifferentiated) and performed duplicate time course experiments. We shut-off endogenous signaling with the TGF $\beta$-receptor kinase inhibitor (SB431542; SB) and then harvested the ESCs for protein and transcriptomic analysis either without any other treatment (T0) or after subsequent treatment with Activin for 3- (T3) or 6-hours (T6). Western blot revealed that SNON and SKI repressors are present at high levels prior to signaling and are degraded upon signaling initiation by Arkadia (Fig. 3A). The RNA-sequencing data have been deposited in NCBI Gene expression Omnibus (accession number: GSE118005).

We found 262 genes with $\geq 2$-fold Activin induction in both WT ESC-lines at T3 (Table S) including several known SMAD2/3 targets(31) are included in this list, providing confidence that they are Activin-induced. 52 of these genes exhibit $\geq 1.7$-fold downregulation in Ark-/- ESCs compered to $W T$ at T3, and were considered as Arkadia-dependent (red font, Table $\mathbf{S}$ ). We then examined the expression of some of these genes in [SnoN/Ark]-/- ESC-lines generated by CRISPR/Cas9, and found that their expression is restored at T3 (Fig. 3B). These genes exhibit $W T$ levels of expression in SnoN-/- ESCs (Fig. S3A) excluding the possibility that there is an over-induction/expression of target genes in the absence of SNON that causes the rescue in [SnoN/Ark]-/- ESCs. This confirms that SNON represses a subset of Activin-induced target genes and that Arkadia removes SNON to allow their expression.

SNON was thought to inhibit broadly SMAD2/3-transcriptional responses because it is recruited to targets by binding to SMAD2, SMAD3 or SMAD4(20). However, the above data show that SNON does not suppress equally all SMAD2/3 target genes. Although it is unknown what determines the susceptibility of target to SNON repression, the data show that highly susceptible 
to SNON repression targets (therefore highly dependent on Arkadia) tend to be silent in the absence of signaling (Fig. S3B). This suggests that the susceptibility to SNON repression is influenced by chromatin and expression potential of targets prior to signaling activation. The finding that SNON is present at high levels in the absence of signaling (Fig. 3A) together with the fact that SMAD4 can bind to SNON and also to the same DNA element as SMAD2/3, suggest that SMAD4-SNON complexes silence SMAD2/3- targets prior to signaling. Furthermore, unlike SMAD2/3, SMAD4 does not have a transactivation domain and cannot recruit co-activators and histone acetylases to its targets, suggesting that SMAD4-SNON are more likely to mediate target gene repression. Recent findings support the above reasoning by showing that SMAD4-SKI represses the master Th17 differentiation gene, ROR $\gamma$ t, which depends on TGF $\beta$ signaling for derepression(32).

Notably, Arkadia-dependent, SMAD2/3 targets are downregulated by $\geq 1$.7-fold in Ark-/- ESCs compared to $W T$ at T3, while at T6 this deficiency is reduced (Fig. 3C and 3D; and Fig. S4A). This is caused by a significant downregulation of several Activin-induced targets in WT ESCs at T6 compared to T3 irrespective of whether they are Arkadia dependent or not, reflecting negative feedback and signaling dynamics. Collectively, the above experiments identified a subset of Activin/NODAL-induced targets susceptible to SNON repression and showed that derepression/activation of these targets by Arkadia is transient and occurs within the first 3 hours of ligand stimulation. 
Fig. 3
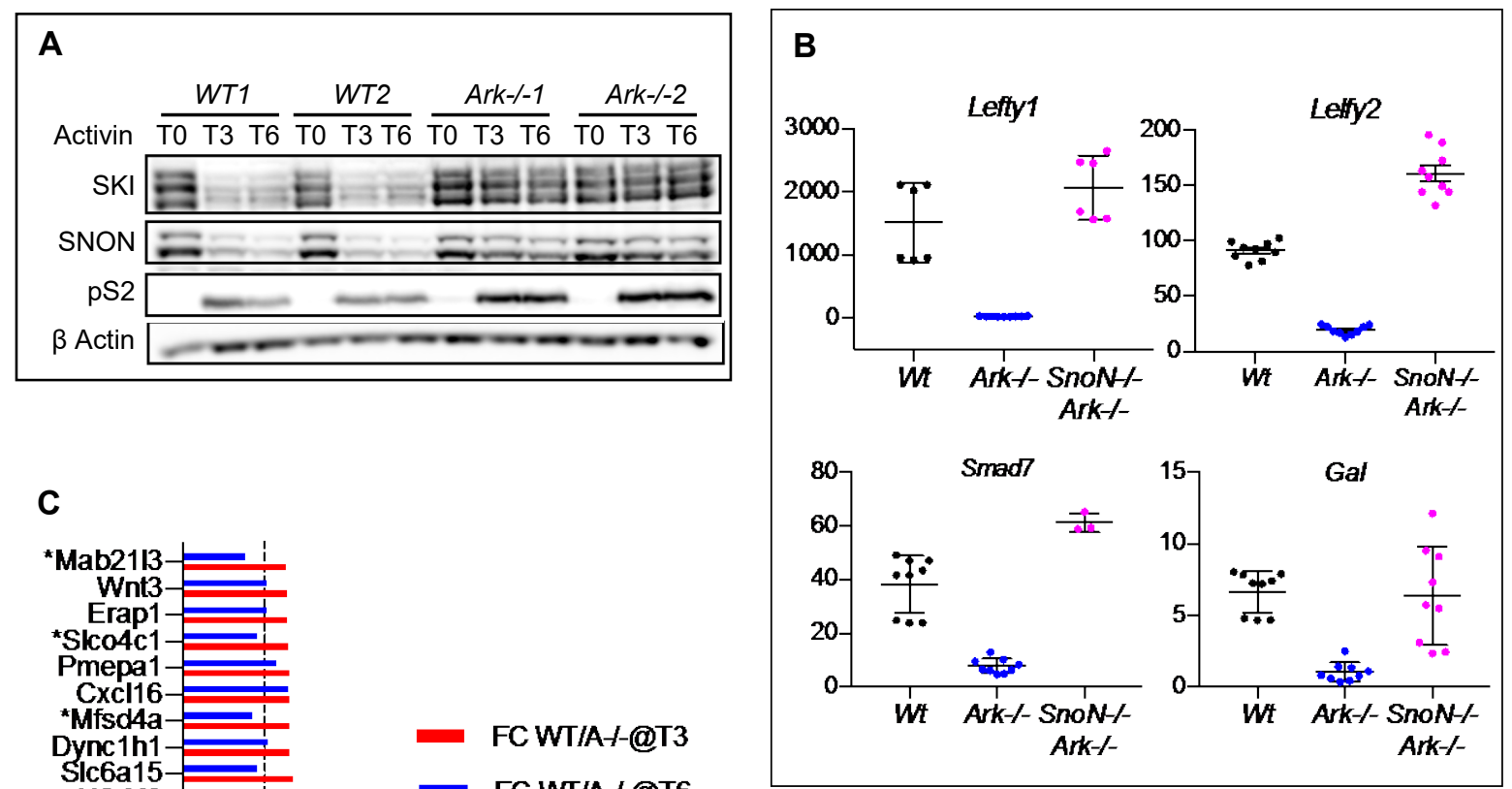

C
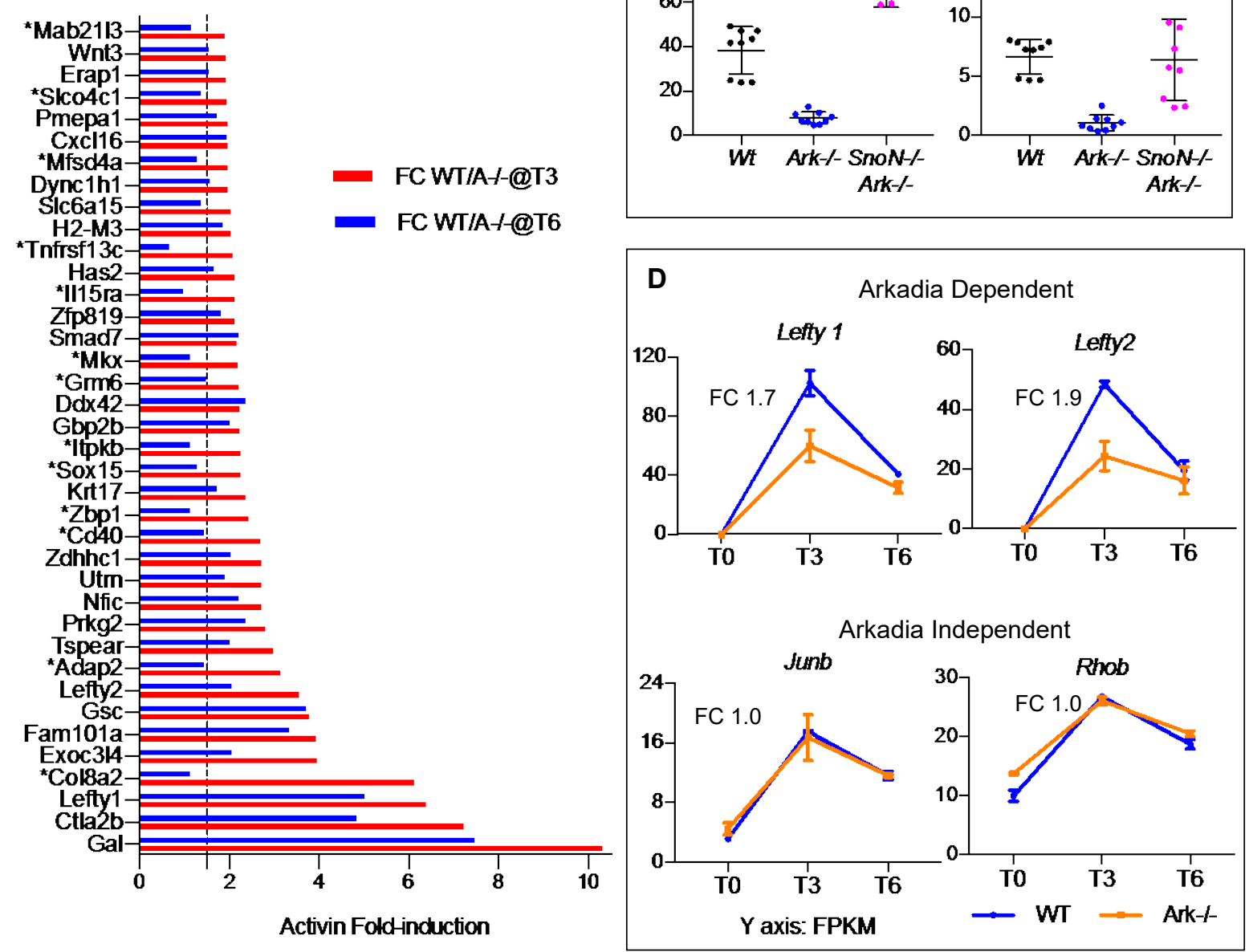

Fig. 3: Arkadia derepresses SNON-repressed genes. (A) Western blot analysis of ESCs treated prior to harvesting for RNA-sequencing. Treatment, time and antibodies are as indicated (pS2: anti-SMAD2). (B) QPCR analysis of representative Arkadia-dependent targets in ESCs with genotype as indicated, pre-treated with SB and then with Activin for 3-hours. (C) A graph comparing Activin induction difference (FC) between $W T$ and Ark-/- ESCs at T3 and T6 of 38 Arkadia-dependent genes (all with $\geq 1$.9-fold activin induction difference between $W T$ and Ark-/- 
ESCs at T3). Asterisks indicate genes showing reduced difference at T6 below $\leq 1.5$-fold difference (dotted line). (D) Expression graphs of representative Arkadia-dependent and independent SMAD2/3-targets from the RNA-sequencing results. The low activation of Arkadiadependent targets at T3 in Ark-/- ESCs is probably due to the mild SNON/SKI reduction observed in (A) by other ubiquitin ligases known to degrade them(20) causing derepression. 


\section{Arkadia degrades SMAD2/3-SNON complexes}

Arkadia is known to function by degrading the repressors SNON and SKI in the presence of TGF $\beta$ signaling(19) and that the interaction of SNON with SMAD2 or SMAD3 is required for SNON degradation(18). Consistent with this in WT and Ark-/- ESCs SNON and SKI are high in the absence of signaling and are degraded within the first hour upon TGF $\beta$-ligand treatment only in WT ESCs but not in Ark-/- (Fig. 4A). Similar to other cell lines(18), SNON levels, unlike those of SKI, recover over time with continuous signaling in WT ESCs. This coincides with the downregulation of the Activin induced transcriptional responses observed in our RNAsequencing experiments at T6 compared to the earlier time of T3 (Fig. 3C and 3D; and Fig. S4A). Unlike $S k i$, SnoN gene is upregulated directly by SMAD2/3 (>3.2-fold; Table S)(33, 34), and this possibly overwhelms Arkadia, which is known to be unstable(14) and therefore contributes to the SNON resistance.

Furthermore, the above experiments showed that in the absence of Arkadia SMAD2, like SNON, accumulates in ESCs (Fig. 3A, Fig. 4A) and embryos (Fig. 4B) possibly in a complex suggesting that Arkadia degrades both. This is supported by our previous study showing that in ESCs Arkadia enhances signaling by interacting directly, ubiquitinating and degrading SMAD2 and SMAD3(14). However, at that time, it was thought that Arkadia degrades the transcriptionally active fraction of SMAD2 which is bound to SMAD4. To address this further, we performed Proximity Ligation Assays (PLA)(35) to visualize SMAD2-SNON (Fig. 4C) and SMAD2SMAD4 (Fig. 4D) complexes in mouse embryonic fibroblasts (MEFs) treated with either SB or with ligand. The data show high accumulation of SMAD2-SNON complexes only in the absence of Arkadia, and equal SMAD2-SMAD4 levels in WT and Ark-/- cells supporting that Arkadia degrades SMAD2-SNON and not SMAD2-SMAD4 complexes. Furthermore, in $W T$ cells ligand 
treatment for one hour seems to be sufficient to eliminates all SMAD2-SNON complexes (Fig.

4C). This suggests that the surge of SMAD2 generated upon signaling initiation pairs with

SNON enabling Arkadia to degrade this complex, leading to derepression/activation of the

SNON repressed targets. However, because SNON becomes resistant to Arkadia over time with signaling degradation and derepression are transient. 
Fig. 4
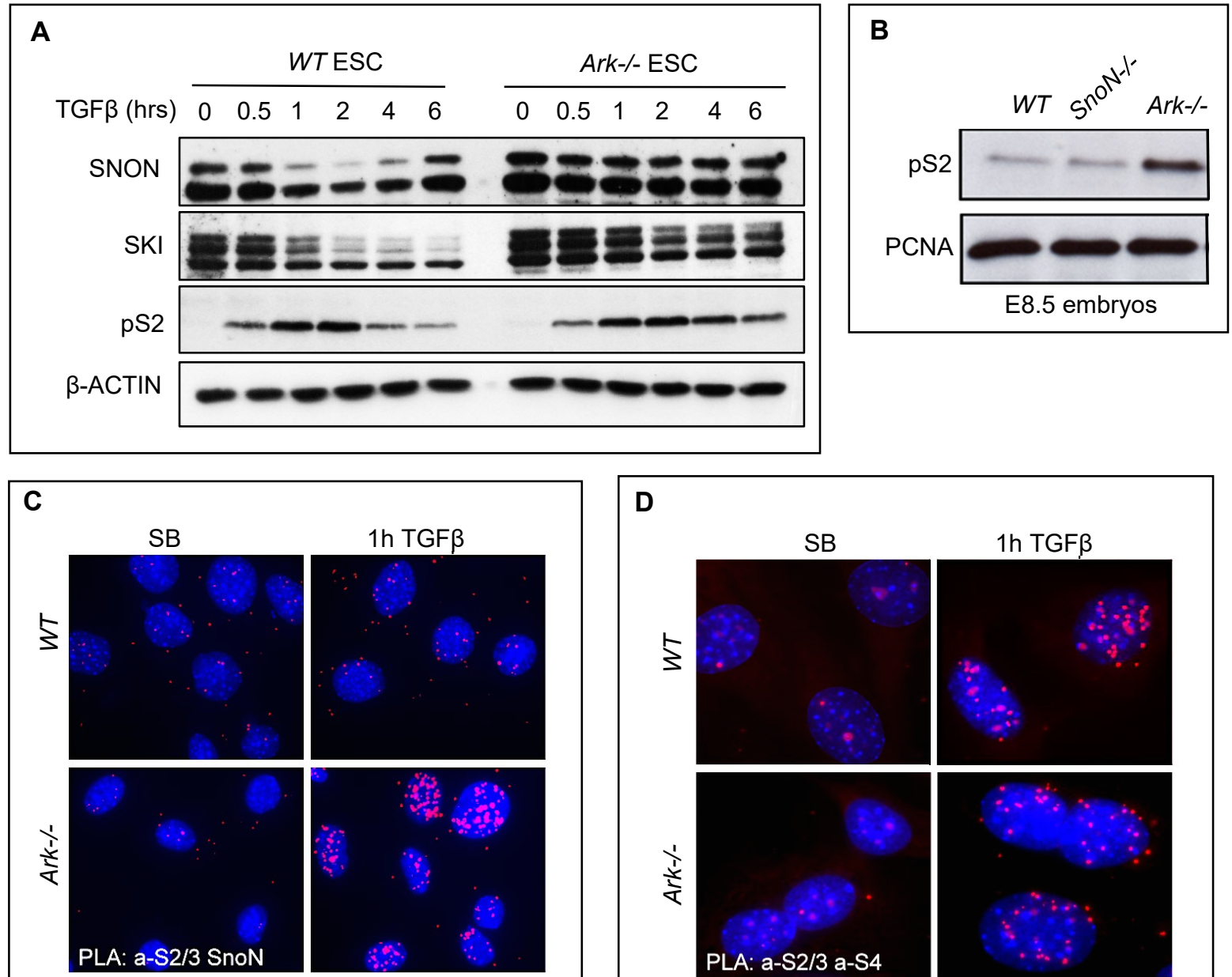

D
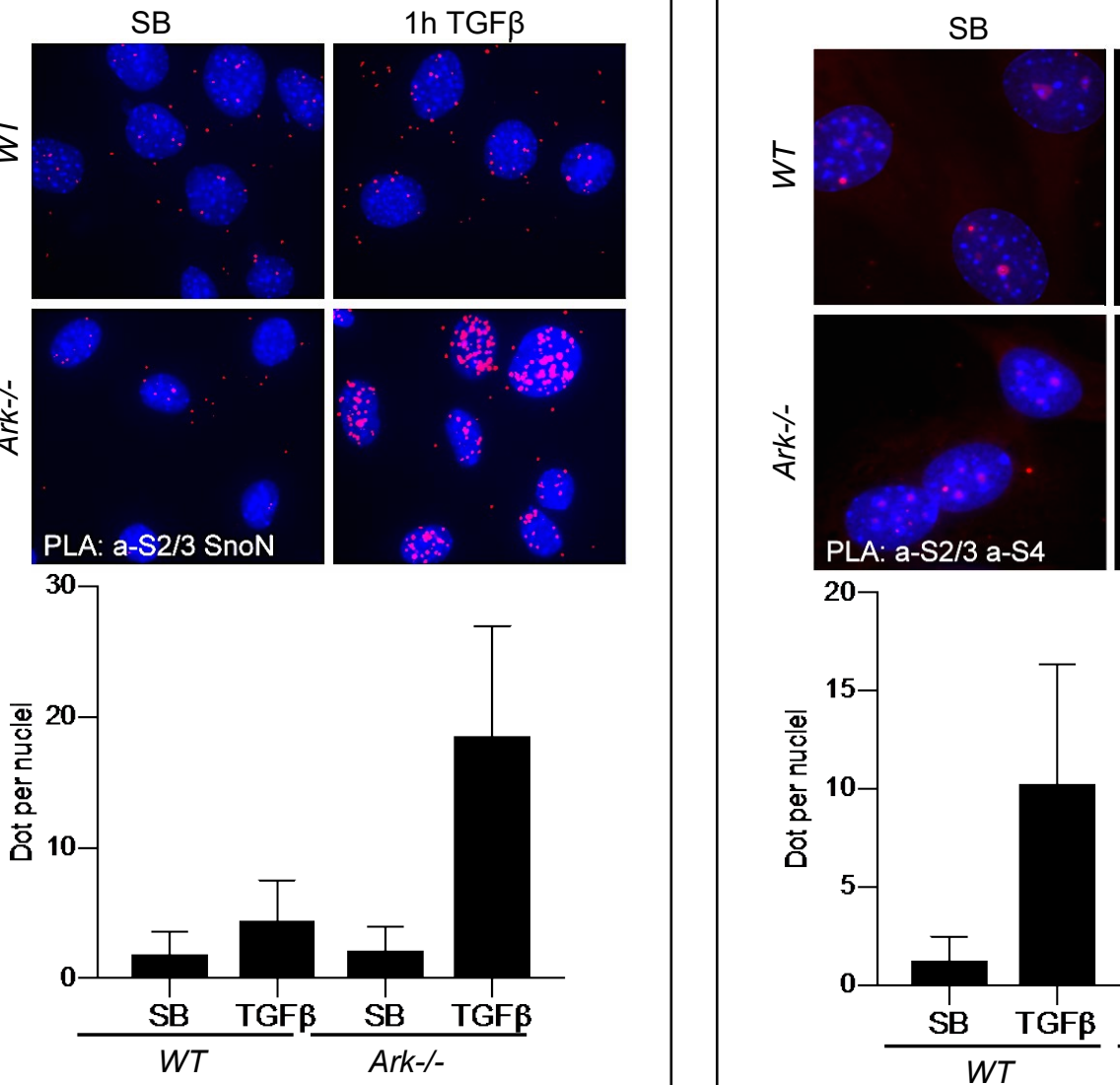

1h TGF $\beta$

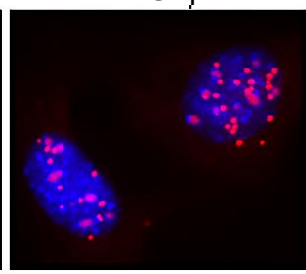

$\frac{1}{\frac{1}{x}}$
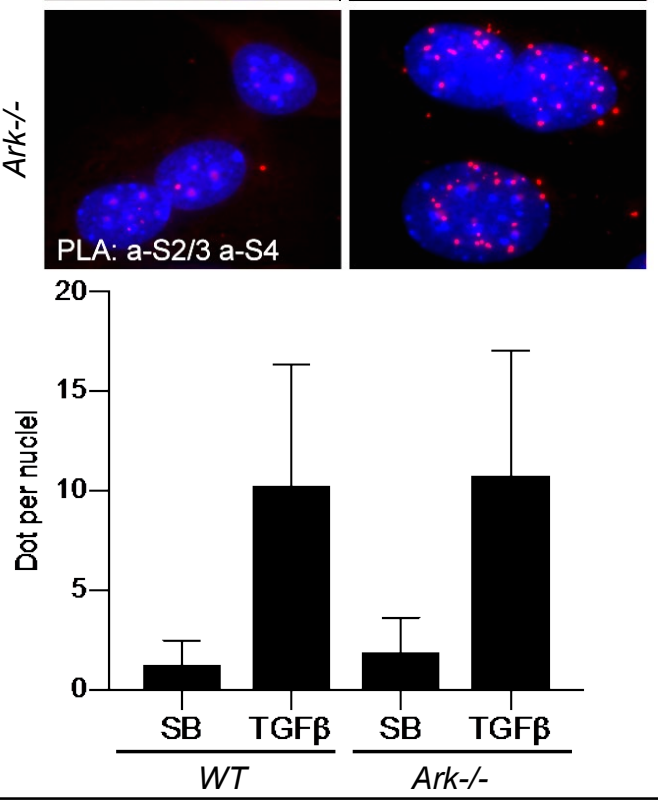
Fig. 4: SMAD2/3 via Arkadia drive SNON degradation. Western blot analysis with antibodies as indicated, on extracts from (A) ESCs treated with TGF $\beta$ ligand over time or SB (“0”) and (B) from embryos with genotypes as shown. The full recovery of SNON levels observed here at 6hrs is not seen in Fig. 3A most likely due to different culturing conditions (see methods). (C and D) Images of PLA experiments (top) and quantitative analysis (bottom) with antibodies, totalSMAD2/3 (S2/3) and SNON in (C), and S2/3 and SMAD4 (S4) in (D). Treatment and genotypes of MEFs are as indicated. 


\section{Signaling intensity drives proportionate SNON reduction and level-dependent responses}

The above pairing of SMAD2-SNON and their degradation by Arkadia seems to perform a comparison of the incoming SMAD2 protein levels relatively to those of SNON which are present in the cell prior to signaling. Therefore, this mechanism most likely provides an assessment of ligand-level exposure and signaling activation for each cell. To investigate whether in the presence of Arkadia the levels of signaling drive proportional SNON-depletion, we assessed SMAD2 and SNON levels downstream specific signaling levels in WT ESCs. To bypass the endogenous expression of various TGF $\beta$ ligands in ESCs, we controlled signaling activation at the receptor level using SB receptor inhibitor. For this we stimulated cells for one hour using fixed amount of Activin (Fig. 5A) or TGF $\beta$ (Fig. S4C) ligands but with different concentration of SB to achieve different levels of signaling-activation. Quantification of the blot showed inverse correlation between SMAD2 and SNON levels with SMAD2:SNON ratio (Fig. 5B) directly proportionate to the levels of signaling. These experiments indicate that in $W T$ ESCs, different signaling levels achieve an equivalent SNON reduction and confirms that SNON is the cellular standard that signaling levels are evaluated on.

To address whether the SMAD2:SNON-protein ratio correlates with the derepression and expression of known SMAD2/3 targets, we used the same Activin/SB treatment and performed qPCR analysis of representative NODAL targets. We found that the NODAL antagonists Leftyl (36) and Lefty2 (37), are not activated under low levels of signaling, and they require maximum signaling for their peak activation (Fig. 5C). Interestingly, they are not expressed at all in the absence of signaling. On the contrary, Smad7 exhibits a low/basal level of expression in the absence of signaling and is upregulated to reach peak expression under moderate levels signaling (Fig. 5C). These results show that each gene exhibits peak response at different SMAD2:SNON- 
protein ratios (compare Fig. 5B and 5C) suggesting variable susceptibility to SNON repression. Therefore, Left 1 is more susceptible to SNON than Lefty2, while Smad7 is mildly suppressed. In the embryo, Leftyl is not normally expressed in the PS but Lefty2 is. WISH showed reduced expression of Lefty2 in Ark-/- embryos (Fig. S5) possibly due to partial derepressed by other ubiquitin ligases such as Smurf2 and APC known to also degrade SNON/SKI(20). This raises the possibility that a subset of SNON/SKI repressed targets is derepressed by these ubiquitin ligases in the absence of Arkadia.

Therefore, SMAD2 levels control SNON-protein reduction via Arkadia leading to derepression and expression of the SNON-repressed NODAL targets in a level-dependent fashion. Furthermore, the variable susceptibility to repression of the NODAL targets most likely leads to unique transcriptional responses downstream of graded signaling. 
Fig. 5

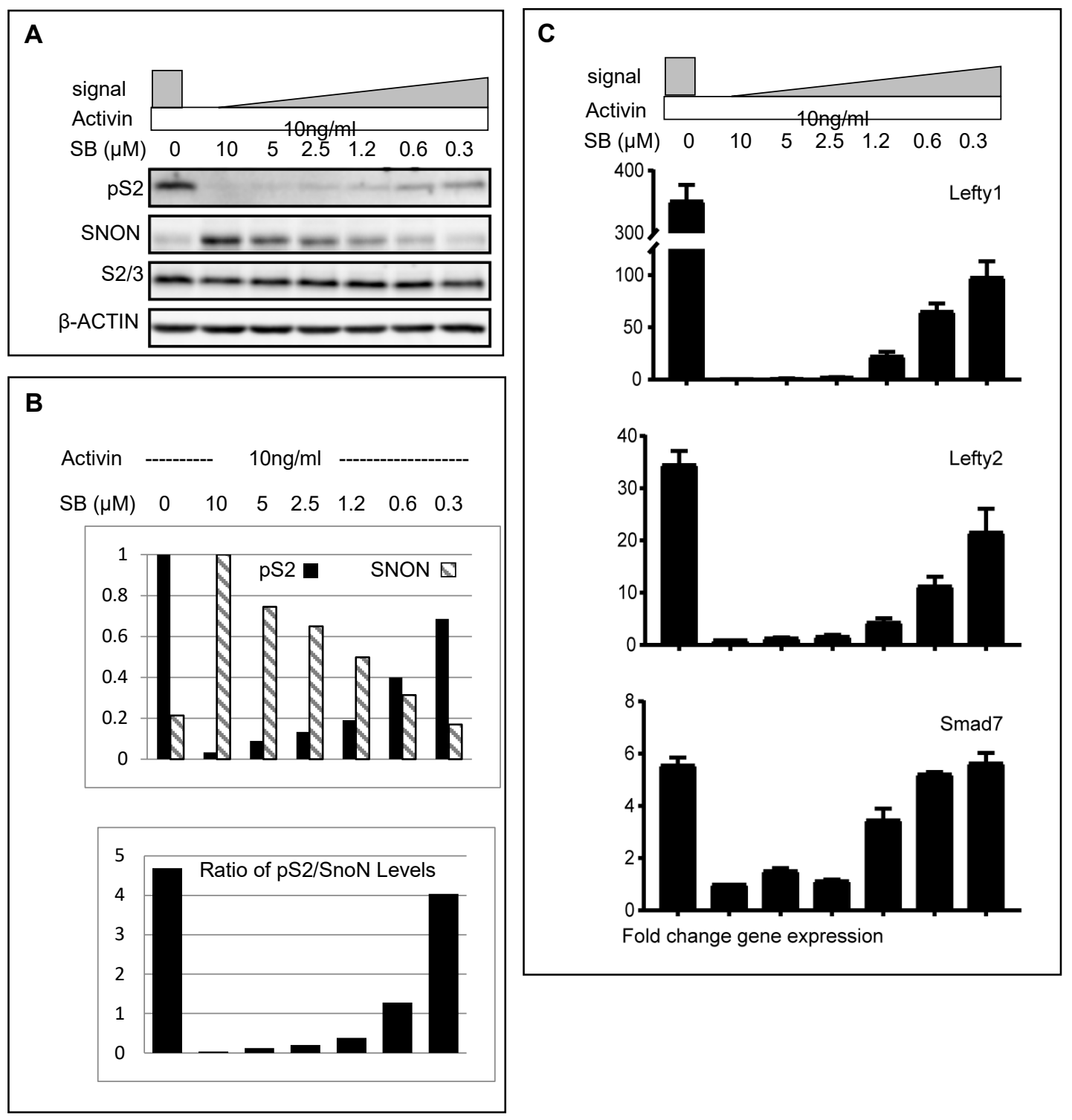

Fig. 5: SMAD2/3 levels drive derepression. (A) Western blot analysis on WT-ESCs treated as indicated for an hour. (B) Quantification of pS2 and SNON proteins (top), and the pS2/SNON ratio (bottom) from the above blot. (C) QPCRs expression analysis for known SMAD2 target genes in WT ESCs treated as indicated for two hours. 


\section{Discussion}

Here we present several lines of evidence including in ESCs and embryos supporting that high NODAL-signaling targets are susceptible to SNON repression and depend on Arkadia and high signaling for SNON removal and derepression. We identified Arkadia-dependent NODALtargets in ESCs and showed that they exhibit variable susceptibility to SNON repression and that their activation occurs under distinct levels of SNON reduction downstream equivalent SMAD2 levels. Therefore, graded NODAL signaling causes variable SNON-reduction and derepression leading to distinct expression patterns.

We showed that SNON becomes resistant to Arkadia over time under continuous NODALligand exposure and that this reinstates target-gene repression in ESCs, suggesting that the initiation of high NODAL-signaling cell-fates, such as ADE, can be reversed with signaling duration. The fact that ADE cells migrate fast out and opposite of the PS and at the same time activate stable expression of Cerberus, a triple antagonist of NODAL, BMP and WNT(38), suggests that the consolidation of $\mathrm{ADE}$ fate requires transient exposure to high signaling. These findings support mounting evidence that derepression and signaling dynamics are instrumental for the interpretation of graded morphogen-signaling(39).

The main principle of the mechanism that interprets graded NODAL-signaling is based on SNON, a SMAD2/3 target-gene repressor and Arkadia a signaling-activated derepressor (Fig. 6). This principle is conserved because the interpretation of the TGF $\beta$ morphogen signaling, DPPMAD, in Drosophila, is controlled by Brinker, a MAD target-gene repressor, and Schnurri, a derepressor, which complexes with activated MAD effectors to suppress Brinker leading to derepression(40-42). Furthermore, Arkadia and SNON are expressed broadly and involved in 
cancer(43-45), suggesting that signaling-dependent derepression and graded transcriptional responses are intrinsic to the TGF $\beta$-SMAD2/3 signaling pathway.

Fig. 6

A

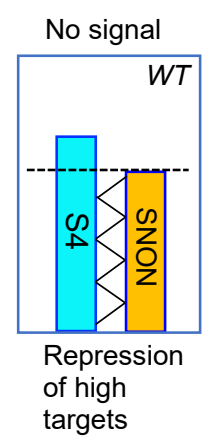

B

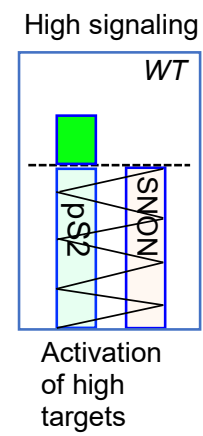

C

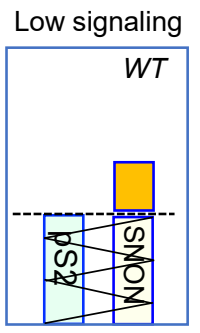

Repression of high targets
D

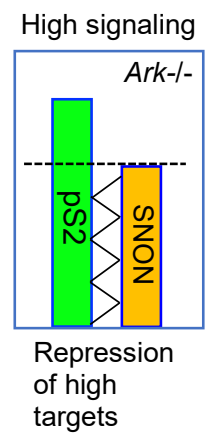

Fig. 6: Signaling-induced derepression controls the activation of high-signaling responses.

Model showing that absence of signaling (A), SNON establish repression via SMAD4 complex formation in a subset of targets. Under high signaling (B), high levels of SMAD2 saturate SNON causing degradation and removal of SNON. This leads to de-repression and activation of repressed targets. Under low signaling $(\mathbf{C})$, SMAD2 reduces SNON causing partial derepression and activation of weakly repressed targets, while highly-repressed targets remain repressed. Absence of Arkadia (D) leads to accumulation of SMAD2-SNON complexes and maintenance of repression on targets. 


\section{References}

1. A. F. Schier, Nodal morphogens. Cold Spring Harb Perspect Biol 1, a003459 (2009).

2. J. Massague, J. Seoane, D. Wotton, Smad transcription factors. Genes Dev 19, 2783-2810 (2005).

3. M. J. Macias, P. Martin-Malpartida, J. Massague, Structural determinants of Smad function in TGF-beta signaling. Trends Biochem Sci 40, 296-308 (2015).

4. C. S. Hill, Transcriptional Control by the SMADs. Cold Spring Harb Perspect Biol 8, (2016).

5. E. H. Budi, D. Duan, R. Derynck, Transforming Growth Factor-beta Receptors and Smads: Regulatory Complexity and Functional Versatility. Trends Cell Biol 27, 658-672 (2017).

6. M. Morikawa, D. Koinuma, K. Miyazono, C. H. Heldin, Genome-wide mechanisms of Smad binding. Oncogene 32, 1609-1615 (2013).

7. E. J. Robertson, Dose-dependent Nodal/Smad signals pattern the early mouse embryo. Semin Cell Dev Biol 32, 73-79 (2014).

8. J. Zinski, B. Tajer, M. C. Mullins, TGF-beta Family Signaling in Early Vertebrate Development. Cold Spring Harb Perspect Biol 10, (2018).

9. K. W. Rogers, A. F. Schier, Morphogen gradients: from generation to interpretation. Annu Rev Cell Dev Biol 27, 377-407 (2011).

10. P. P. Tam, R. R. Behringer, Mouse gastrulation: the formation of a mammalian body plan. Mech Dev 68, 3-25 (1997).

11. N. R. Dunn, S. D. Vincent, L. Oxburgh, E. J. Robertson, E. K. Bikoff, Combinatorial activities of Smad2 and Smad3 regulate mesoderm formation and patterning in the mouse embryo. Development 131, 1717-1728 (2004). 
12. V. Episkopou et al., Induction of the mammalian node requires Arkadia function in the extraembryonic lineages. Nature 410, 825-830 (2001).

13. C. Niederlander, J. J. Walsh, V. Episkopou, C. M. Jones, Arkadia enhances nodal-related signalling to induce mesendoderm. Nature 410, 830-834 (2001).

14. K. J. Mavrakis et al., Arkadia enhances Nodal/TGF-beta signaling by coupling phosphoSmad2/3 activity and turnover. PLoS Biol 5, e67 (2007).

15. D. Koinuma et al., Arkadia amplifies TGF-beta superfamily signalling through degradation of Smad7. EMBO J 22, 6458-6470 (2003).

16. P. Kavsak et al., Smad7 binds to Smurf2 to form an E3 ubiquitin ligase that targets the TGF beta receptor for degradation. Mol Cell 6, 1365-1375 (2000).

17. A. Nakao et al., Identification of Smad7, a TGFbeta-inducible antagonist of TGF-beta signalling. Nature 389, 631-635 (1997).

18. L. Levy et al., Arkadia activates Smad3/Smad4-dependent transcription by triggering signal-induced SnoN degradation. Mol Cell Biol 27, 6068-6083 (2007).

19. Y. Nagano et al., Arkadia induces degradation of SnoN and c-Ski to enhance transforming growth factor-beta signaling. J Biol Chem 282, 20492-20501 (2007).

20. J. Deheuninck, K. Luo, Ski and SnoN, potent negative regulators of TGF-beta signaling. Cell Res 19, 47-57 (2009).

21. M. Rothova, J. J. Holzenspies, A. Livigni, S. N. Villegas, J. M. Brickman, Differentiation of Mouse Embryonic Stem Cells into Ventral Foregut Precursors. Curr Protoc Stem Cell Biol 36, $1 \mathrm{G} 3$ 1-12 (2016). 
22. T. A. Rodriguez, E. S. Casey, R. M. Harland, J. C. Smith, R. S. Beddington, Distinct enhancer elements control Hex expression during gastrulation and early organogenesis. Dev Biol 234, 304-316 (2001).

23. G. M. Morrison et al., Anterior definitive endoderm from ESCs reveals a role for FGF signaling. Cell Stem Cell 3, 402-415 (2008).

24. S. L. Ang et al., The formation and maintenance of the definitive endoderm lineage in the mouse: involvement of HNF3/forkhead proteins. Development 119, 1301-1315 (1993).

25. R. M. Arkell, P. P. Tam, Initiating head development in mouse embryos: integrating signalling and transcriptional activity. Open Biol 2, 120030 (2012).

26. S. Pearson-White, M. McDuffie, Defective T-cell activation is associated with augmented transforming growth factor Beta sensitivity in mice with mutations in the Sno gene. Mol Cell Biol 23, 5446-5459 (2003).

27. J. A. Belo et al., Cerberus-like is a secreted factor with neutralizing activity expressed in the anterior primitive endoderm of the mouse gastrula. Mech Dev 68, 45-57 (1997).

28. M. Tojo et al., Smad7-deficient mice show growth retardation with reduced viability. $J$ Biochem 151, 621-631 (2012).

29. G. Oliver et al., Six3, a murine homologue of the sine oculis gene, demarcates the most anterior border of the developing neural plate and is expressed during eye development. Development 121, 4045-4055 (1995).

30. Y. Echelard et al., Sonic hedgehog, a member of a family of putative signaling molecules, is implicated in the regulation of CNS polarity. Cell 75, 1417-1430 (1993).

31. M. Guzman-Ayala et al., Graded Smad2/3 activation is converted directly into levels of target gene expression in embryonic stem cells. PLoS One 4, e4268 (2009). 
32. S. Zhang et al., Reversing SKI-SMAD4-mediated suppression is essential for TH17 cell differentiation. Nature 551, 105-109 (2017).

33. Q. Zhu, K. Luo, SnoN in regulation of embryonic development and tissue morphogenesis. FEBS Lett 586, 1971-1976 (2012).

34. T. Gaarenstroom, C. S. Hill, TGF-beta signaling to chromatin: how Smads regulate transcription during self-renewal and differentiation. Semin Cell Dev Biol 32, 107-118 (2014).

35. E. Thymiakou, V. Episkopou, Detection of signaling effector-complexes downstream of bmp4 using PLA, a proximity ligation assay. J Vis Exp, (2011).

36. C. Meno et al., lefty-1 is required for left-right determination as a regulator of lefty-2 and nodal. Cell 94, 287-297 (1998).

37. C. Meno et al., Mouse Lefty2 and zebrafish antivin are feedback inhibitors of nodal signaling during vertebrate gastrulation. Mol Cell 4, 287-298 (1999).

38. S. Piccolo et al., The head inducer Cerberus is a multifunctional antagonist of Nodal, BMP and Wnt signals. Nature 397, 707-710 (1999).

39. A. Sagner, J. Briscoe, Morphogen interpretation: concentration, time, competence, and signaling dynamics. Wiley Interdiscip Rev Dev Biol 6, (2017).

40. G. Campbell, A. Tomlinson, Transducing the Dpp morphogen gradient in the wing of Drosophila: regulation of Dpp targets by brinker. Cell 96, 553-562 (1999).

41. A. Jazwinska, N. Kirov, E. Wieschaus, S. Roth, C. Rushlow, The Drosophila gene brinker reveals a novel mechanism of Dpp target gene regulation. Cell 96, 563-573 (1999). 
42. M. Minami, N. Kinoshita, Y. Kamoshida, H. Tanimoto, T. Tabata, brinker is a target of Dpp in Drosophila that negatively regulates Dpp-dependent genes. Nature 398, 242-246 (1999).

43. V. Sharma et al., Enhancement of TGF-beta signaling responses by the E3 ubiquitin ligase Arkadia provides tumor suppression in colorectal cancer. Cancer Res 71, 6438-6449 (2011).

44. E. Le Scolan et al., Transforming growth factor-beta suppresses the ability of Ski to inhibit tumor metastasis by inducing its degradation. Cancer Res 68, 3277-3285 (2008).

45. M. A. Briones-Orta et al., Arkadia regulates tumor metastasis by modulation of the TGFbeta pathway. Cancer Res 73, 1800-1810 (2013).

\section{Acknowledgments}

We wish to thank Prof. Susumu Itoh (Showa Pharmaceutical Univ. Tokyo) for donating the Smad7 KO mice and Prof. Kunxin Luo (U.C. Berkeley) for the SnoN KO mice. We thank Dr. Vikas Sharma, Michael Normal and Nabiha Husain for preliminary experiments. Drs. Tristan Rodriguez and Aida DiGregorio (ICL) for help with in-situ hybridizations, Eleni Ioannou (ICL), Zoe Webster (MRC/ICL) and Dr. Christophe Galichet (Crick Institute) for technical assistance and animal procedures. We also thank Prof. Kunxin Luo for critical reading of the MS and suggestions.

Funding: This work was funded by the MRC UK grant (MR/M011194). 
Authors contributions: J.M.C. and M.I. contributed equally by designing, conducting and analyzing experiments; and by providing a draft of figures, methods and results. VE raised funding; designed and conducted experiments; interpreted the combined results; and wrote the manuscript.

Competing interests: The authors declare no competing interests.

Data and materials availability: The RNA sequencing data have been deposited in NCBI Gene expression Omnibus (accession number GSE118005).

\section{List of Supplementary Materials}

Materials and Methods

Fig S1 - S5

\section{Other Supplementary Materials for this manuscript include the following:}

Caption: Table $\mathbf{S}$ (see Table $\mathbf{S}$ containing the list of Activin-induced genes in the Auxiliary supplementary data). 